\title{
Lactobacillus fermentum Ess-1 with unique growth inhibition of vulvo-vaginal candidiasis pathogens
}

\author{
Correspondence \\ Daniel Rönnqvist \\ daniel.ronnqvist@essum.se
}

Received 12 February 2007

Accepted 3 July 2007

\section{Daniel Rönnqvist, ${ }^{1}$ Ulla Forsgren-Brusk, ${ }^{2}$ Ulrika Husmark ${ }^{2}$} and Eva Grahn-Håkansson ${ }^{3}$

\author{
${ }^{1}$ Department of Clinical Bacteriology, Umeå University, Umeå, Sweden \\ ${ }^{2}$ SCA Hygiene Products AB, Gothenburg, Sweden \\ ${ }^{3}$ Essum $A B$, Umeå, Sweden
}

\section{INTRODUCTION}

Vulvo-vaginal candidiasis (VVC) is characterized by overgrowth of yeast species in the vulva and vagina. Candida albicans accounts for $90 \%$ of all VVC infections, and other yeast species causing VVC are often categorized as non-albicans species (Paulitsch et al., 2006). An increased prevalence of non-albicans species, foremost Candida glabrata, has been detected in recent years and has led to increased medical concern (Martens et al., 2004; Spinillo et al., 1997). VVC caused by C. glabrata is more difficult to diagnose by microscopy because of the lack of hyphae, and needs to be verified by culture. There is also no clear strategy regarding the treatment of VVC caused by this species. C. glabrata has low susceptibility to azoles, increasing the need for new treatment strategies (Sobel et al., 2003).

Lactobacilli are natural habitants of the vulvo-vaginal microflora and are believed to have a central role in the suppression of potential pathogens. Lactobacilli administered to the genital tract have a prominent role as a prophylactic aimed at improving the genital microfloral defence against bacterial infections (Juarez Tomas et al., 2003; Kaewsrichan et al., 2006; Voravuthikunchai et al., 2006). Strains that exert a health benefit to the host are classified as probiotics. Probiotic strains that can be used for the treatment of VVC should be able to produce

Abbreviations: $L A B$, lactic acid bacteria; LCF, Lactobacillus cell-free filtrate; VVC, vulvo-vaginal candidiasis. metabolites that are fungistatic for $C$. albicans and $C$. glabrata. Results where metabolites from Lactobacillus isolates strongly affect Candida growth in vitro have only been published in a few reports and relate to C. albicans in particular (Okkers et al., 1999; Strus et al., 2005b; Wynne et al., 2004). To the best of our knowledge, no studies have demonstrated an effect of Lactobacillus isolates on the growth of C. glabrata. The aim of the present study was to find a probiotic candidate that inhibited the growth of both C. albicans and C. glabrata.

\section{METHODS}

Bacteria and yeast isolates. A total of 126 human Lactobacillus isolates was isolated from the forehead, throat and teeth of healthy adult volunteers and from faecal samples from newborn infants obtained 3-5 days after birth. Swab samples obtained from the donors were cultured on a Lactobacillus-selective agar [de Man, Rogosa and Sharpe (MRS) agar; Merck] at $37{ }^{\circ} \mathrm{C}$ for $48 \mathrm{~h}$ with $5 \% \mathrm{CO}_{2}$.

All isolates were screened for yeast growth inhibiting capacity. The four target Candida isolates were vaginal isolates from women with VVC (C. albicans 702, C. albicans A, C. glabrata 1 and C. glabrata 2). In addition, two reference strains (C. albicans CCUG 44135 and $C$. glabrata CCUG 44136), originally isolated from the vagina of healthy females, were used. In screening procedures, the previously characterized strain Lactobacillus plantarum LB931 (Rönnqvist et al., 2005) was used as a reference, together with additional Lactobacillus strains with various fungistatic properties. These reference strains were Lactobacillus casei Shirota (Yakult), L. casei Defensis (Danone), Lactobacillus fermentum ITM6E, Lactobacillus brevis ITM1F (Microbiology Institute of the Catholic University of Piacenza, 
Italy), Lactobacillus rhamnosus IMPC19 (both from the Institute of Sciences of Food Production, CNR, Bari, Italy), L. rhamnosus GG (ATCC 53103) and L. plantarum 299v (DSM 9843). L. fermentum LB99 is an isolate that does not produce metabolites that inhibit the growth of Candida and hence was used as a negative control in the experiment assessing the effect of $\mathrm{pH}$ on inhibition described below.

Initial screening using a modified agar overlay technique. Each Lactobacillus isolate was cultured in MRS broth (Merck) for $20 \mathrm{~h}$ at $37^{\circ} \mathrm{C}$. The broth was then added to wells in a Bertani tray and stamped onto an MRS agar plate using a sterile Steer's steel pin replicator. The agar plate was incubated under anaerobic conditions at $37{ }^{\circ} \mathrm{C}$ for $24 \mathrm{~h}$. Thereafter, $12 \mathrm{ml}$ Sabouraud dextrose agar (LAB M) was poured onto the MRS agar and allowed to solidify. Approximately $6 \log _{10} C$. albicans organisms were seeded onto the Sabouraud dextrose agar and the plate was incubated aerobically at $37{ }^{\circ} \mathrm{C}$ for $24 \mathrm{~h}$. Candida growth inhibition on the spot located above each Lactobacillus isolate was evaluated, and Lactobacillus isolates inhibiting growth equal to or greater than L. plantarum LB931 were selected for further secondary screening.

Secondary screening by cell-free filtrate inhibition. Each Lactobacillus isolate was cultured in $4 \mathrm{ml}$ dMRSs broth (MRS broth without the addition of sodium acetate; Stiles et al., 2002) for $20 \mathrm{~h}$ at $37{ }^{\circ} \mathrm{C}$ and then centrifuged at $1900 \mathrm{~g}$ for $10 \mathrm{~min}$ at $10{ }^{\circ} \mathrm{C}$. The supernatant was sterilized by passing through a $0.22 \mu \mathrm{m}$ pore-size filter and the $\mathrm{pH}$ was monitored using a $\mathrm{pH}$ meter (Metrohm). This filtrate was designated Lactobacillus cell-free filtrate (LCF). Aliquots $(750 \mu \mathrm{l})$ of LCF were transferred to the wells of a 48 -well microtitre plate (Sarstedt), air-dried at $45{ }^{\circ} \mathrm{C}$ for $20 \pm 2 \mathrm{~h}$ and resuspended in $250 \mu \mathrm{l}$ sterile distilled water. A total of $200 \mu \mathrm{l}$ of each concentrated LCF was transferred to a well of a 96-well microtitre plate. Controls contained fresh dMRSs or dMRSs broth with the $\mathrm{pH}$ adjusted to the same $\mathrm{pH}$ as the lowest monitored LCF $\mathrm{pH}$, and otherwise treated as the LCF samples. Three isolates each of C. albicans and C. glabrata were inoculated in the wells at a final concentration of $4.5 \log _{10}$ c.f.u. $\mathrm{ml}^{-1}$ and grown at $37^{\circ} \mathrm{C}$. After $24 \mathrm{~h}$, the growth inhibition score was determined according to the scale shown in Fig. 1. Inhibition was scored from 0 to 10 , where LCFs with lack of inhibition equal to the controls were assigned a score of 0 , and LCFs showing complete inhibition of Candida growth were assigned a score of 10. All samples were analysed in duplicate and the inhibition score was determined by two independent investigators who were blind to the origin of the sample. The inhibition assay was repeated twice and the results of each isolate were presented as the mean of the determined values.

API typing and genetic typing of Lactobacillus isolates. Identification to the species level was carried out using the API 50 CHL system (bioMérieux), following the manufacturer's instructions. Data from the fermentation tests were analysed using API LAB PLUS software. Genetic typing was carried out by the Deutsche Sammlung von Mikroorganismen und Zellkulturen GmbH (DSMZ, Gent, Belgium) by partial sequence analysis of the 16S rRNA gene.

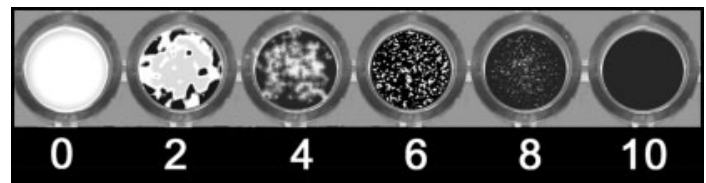

Fig. 1. Determination of Candida growth inhibition scores. No visual inhibition was scored as 0 and complete visual inhibition as 10 .
Effect of pH on inhibition with LCF. The $\mathrm{pH}$ of two LCFs was adjusted to 5.0 and 7.0 using $2 \mathrm{M}$ sodium hydroxide. A third test tube containing LCF at the original $\mathrm{pH}$ of 3.7 was not adjusted. The LCFs were stabilized at $+8{ }^{\circ} \mathrm{C}$ for $1 \mathrm{~h}$ and centrifuged at $2480 \mathrm{~g}$ at $10{ }^{\circ} \mathrm{C}$ for $15 \mathrm{~min}$. The supernatant was sterilized through a $0.22 \mu \mathrm{m}$ poresize sterile filter and the filtrate was transferred to 96 -well microtitre plates. C. albicans CCUG 44135 was added to a final concentration of $4.5 \log _{10}$ c.f.u. $\mathrm{ml}^{-1}$. The plates were incubated at $37^{\circ} \mathrm{C}$ for $24 \mathrm{~h}$ and visually evaluated by two investigators as described in the secondary screening process. All samples were analysed in duplicate.

Survival following freeze-drying and storage at $37{ }^{\circ} \mathrm{C} . L$. fermentum Ess-1 was cultured in MRS broth for $18 \mathrm{~h}$ at $37^{\circ} \mathrm{C}$, centrifuged at $3100 \mathrm{~g}$ at $6{ }^{\circ} \mathrm{C}$ for $22 \mathrm{~min}$ and the pellet concentrated tenfold in $20 \%$ trehalose (Bröste). The suspension was frozen at $-55{ }^{\circ} \mathrm{C}$ and thereafter freeze-dried in a Hetosicc freeze-drier (Heto Birkerød) for $48 \mathrm{~h}$. The end product was pulverized with a mortar and stored in a sealed plastic tube at $37^{\circ} \mathrm{C}$ for 11 weeks. The number of viable bacteria was determined at weeks $0,2,5$ and 11 .

Vaginal insertion of $\boldsymbol{L}$. fermentum Ess-1. Lyophilized powder containing L. fermentum Ess-1 was placed into gelatin capsules (size 2; Apoteket) at a concentration of $\sim 10^{9}$ c.f.u. per capsule. Four healthy, pre-menopausal volunteers (aged 19-28 years) introduced one capsule to the vagina 1 week prior to menses. Vaginal samples were taken prior to vaginal insert and the day after the last bleeding day by turning a sterile cotton swab three times $3 \mathrm{~cm}$ above the introitus. The cotton swab was stored in MRS broth at $8{ }^{\circ} \mathrm{C}$ for not more than $48 \mathrm{~h}$ until quantification of $L$. fermentum Ess- 1 . The sample was cultured on Rogosa agar plates with $128 \mathrm{mg}$ vancomycin $1^{-1}$ to minimize the growth of endogenous lactobacilli. Colonies were counted and the number of c.f.u. $\mathrm{ml}^{-1}$ was determined. The study was approved by the Regional Ethical Review Board in Umeå, Sweden.

Susceptibility to antifungal drugs. The susceptibility of $L$. fermentum Ess-1 to antifungal substances was tested using E-tests (AB Biodisk) with amphotericin, caspofungin, fluconazole, flucytocine, itraconazole, ketoconazole, posaconazole and voriconazole. A total of $100 \mu \mathrm{L}$. fermentum Ess- 1 and $0.9 \%$ sodium chloride suspension corresponding to $1 \mathrm{McFarland}$ standard was seeded onto agar plates containing Iso-Sensitest agar (Oxoid) with the addition of $5 \%$ horse blood. After $15 \mathrm{~min}$, the E-tests were applied to the agar and the plates were incubated for $24 \mathrm{~h}$ with $5 \% \mathrm{CO}_{2}$ at $37{ }^{\circ} \mathrm{C}$. The susceptibility results were determined according to the manufacturer's instructions.

Growth inhibition effect on vaginal lactic acid bacteria (LAB) isolates. A previously described method (Rönnqvist et al., 2005) was carried out, with some modifications, to evaluate whether metabolites produced by L. fermentum Ess-1 inhibited the growth of other LAB. Briefly, L. fermentum Ess-1 was inoculated into a $25 \mathrm{ml}$ modified MRS agar layer (sodium acetate concentration adjusted to $0.29 \mathrm{M}$ ) to a final concentration of $7.5 \log _{10}$ c.f.u. $\mathrm{ml}^{-1}$. The plate was incubated for $24 \mathrm{~h}$ and an additional layer of MRS agar with the addition of $\mathrm{KH}_{2} \mathrm{PO}_{4}$ (final concentration $0.17 \mathrm{M}$ ) was poured on top of the first MRS agar layer. Ten LAB isolates [L. plantarum (4), L. rhamnosus (1), Lactobacillus paracasei (1), Lactobacillus cellobiosus (1), Pediococcus pentosaceus (2) and Pediococcus acidilactici (1)] were stamped onto the solidified and dried agar using a Steer's steel-pin replicator. The plate was incubated at $37{ }^{\circ} \mathrm{C}$ in $5 \% \mathrm{CO}_{2}$ for $24 \mathrm{~h}$. The results were assessed as 'inhibition' (no or weak visual growth) or 'no inhibition' (strong growth).

\section{RESULTS AND DISCUSSION}

Use of probiotic bacteria for the treatment of disorders of the urogenital sphere has focused mainly on urinary tract 
infections and bacterial vaginosis. The possibility of preventing VVC using probiotics has been less extensively investigated (Reid \& Bruce, 2003). In bacterial vaginosis, there is always a quantitative reduction in the number of endogenous vaginal lactobacilli. Trials aiming to restore dominance of the Lactobacillus flora by oral and/or local application of probiotic strains have not been conclusive, indicating that not only quantitative but also qualitative properties of the normal flora are essential (Wilson, 2004). In VVC, there is no such clear relationship between Candida overgrowth and paucity of lactobacilli in the vaginal flora (Demirezen, 2002). Given the clinical impact of VVC, alternative treatment strategies for this condition should be explored. Qualitative properties, such as inhibition of yeast growth, could be an important feature of a candidate probiotic strain for the treatment of VVC.

To determine a probiotic strategy for the treatment of VVC, we focused our search on Lactobacillus strains that produced metabolites that inhibited the growth of Candida species. We suggest that promising probiotic bacteria should be evaluated with respect to growth inhibition of both $C$. albicans and C. glabrata, as these are the most frequently isolated VVC pathogens. To the best of our knowledge, only one in vitro study regarding the growth inhibitory effect of lactobacilli on these Candida species has been published (Strus et al., 2005a), where 25 vaginal Lactobacillus isolates of different species were studied. Isolates typed as Lactobacillus delbrueckii exhibited the strongest inhibitory activity against $C$. albicans, but none of the isolates showed growth inhibition activity against $C$. glabrata.

\section{Screening and typing}

Previous work from our own laboratory has shown a total lack of $C$. albicans growth inhibition by vaginal Lactobacillus isolates (Rönnqvist et al., 2005). Those findings taken together with the findings of Strus et al. (2005a) were used to design the present study encompassing the screening of Lactobacillus strains isolated from a number of different sites in order to maximize variation in the properties among strains. The 126 human Lactobacillus strains used were isolated from teeth $(n=82)$, throat $(n=5)$, forehead $(n=3)$ and neonatal faeces $(n=36)$. In the initial screening process, 18/126 Lactobacillus isolates $(14.3 \%)$, the majority of which were collected from the oral tract of healthy adults, showed a detectable inhibitory effect on C. albicans growth comparable to or exceeding that of L. plantarum LB931. In addition, C. albicans inhibition was found in the two reference strains $L$. rhamnosus IMPC19 and L. rhamnosus GG. These 20 Lactobacillus isolates, together with L. plantarum LB931, were selected for secondary screening. Fig. 2 illustrates the growth inhibition scores for each Lactobacillus isolate against three different $C$. albicans isolates and three different C. glabrata isolates. Of the 21 Lactobacillus isolates tested in the secondary screening, only 1 proved

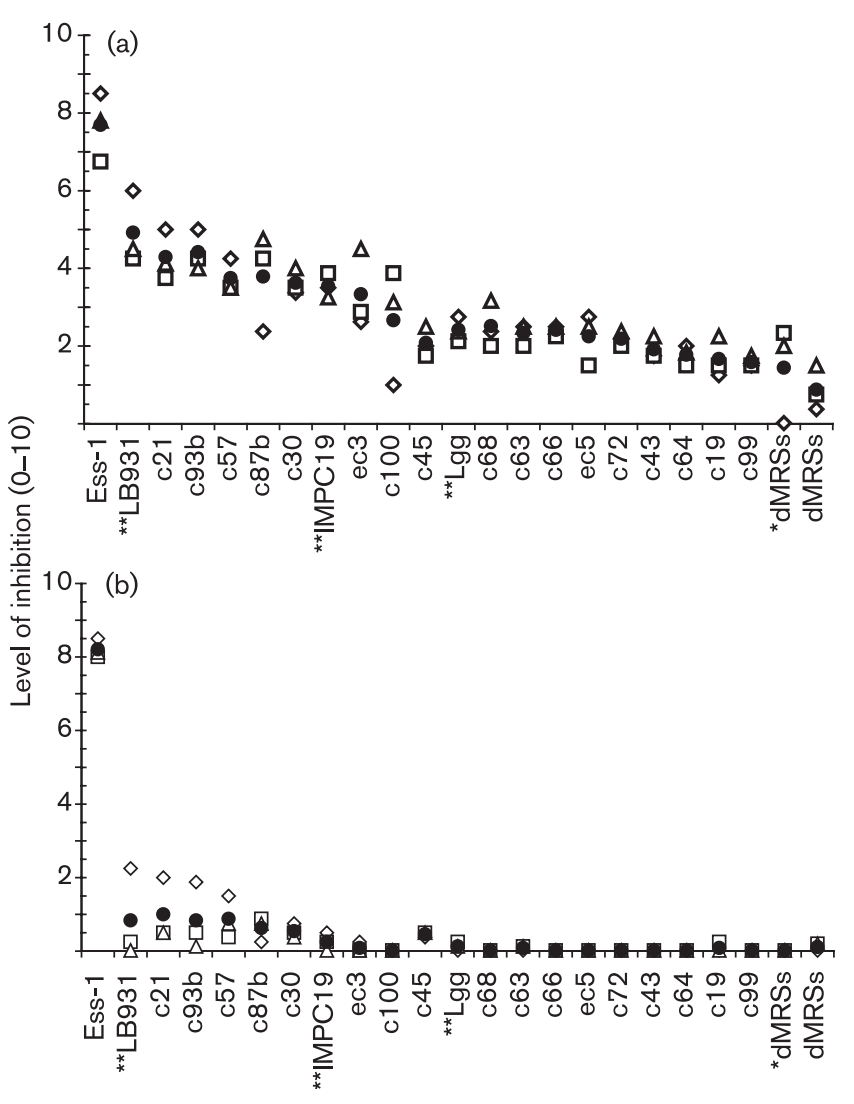

Fig. 2. Secondary screening inhibition scores for LCF against $C$. albicans (a) and C. glabrata (b). C. albicans or C. glabrata isolates were inoculated into LCFs from different Lactobacillus isolates. After $24 \mathrm{~h}$, the growth inhibition score for each Lactobacillus isolate was determined according to Fig. 1 on a scale of $0-10$. The break point of each Candida isolate is shown as follows: (a) $\diamond, C$. albicans CCUG 44135; $\square$, C. albicans 702; $\triangle$, C. albicans A; and (b) $\diamond$, C. glabrata CCUG 44136; $\square$, C. glabrata $1 ; \triangle$, C. glabrata 2. - The mean of all three isolates; ${ }^{*}$, dMRSs broth with $\mathrm{pH}$ adjusted to the lowest monitored $\mathrm{pH}$ of all filtrates $(\mathrm{pH} 3.8) ;{ }^{* *}$, reference strains.

to have a strong inhibitory activity against both Candida species. This strain was originally isolated from the throat of a healthy person and was typed as L. fermentum. It was designated L. fermentum Ess-1 and is the first Lactobacillus strain described with significant growth inhibition activity against both C. albicans and C. glabrata.

\section{Initial characterization of L. fermentum Ess-1}

Studies were carried out to evaluate the potential probiotic use of L. fermentum Ess-1 in vaginal health. As such, the stability of the metabolites inhibiting Candida growth at different vaginal $\mathrm{pH}$ levels was of interest. The vaginal $\mathrm{pH}$ among healthy women, as well as in women with VVC, is normally around 4.5 (Sobel \& Chaim, 1996), but varies among individuals. No sign of decreased activity in $\mathrm{pH}-$ adjusted filtrate between $\mathrm{pH} 3.7$ and 7.0 was found 
(Table 1), indicating that the active metabolites were stable within the clinically relevant $\mathrm{pH}$ range that occurs in the vagina and on vulvar skin (Runeman et al., 2004).

Furthermore, probiotic lactobacilli should not interfere with the normal vulvo-vaginal LAB flora. Nine out of ten tested LAB were not inhibited by L. fermentum Ess-1 (not shown). Only one strain of L. cellobiosus, a rather rare isolate of the vaginal flora (Vasquez et al., 2002), was affected by metabolites produced by L. fermentum Ess-1.

Kilic et al. (2005) studied the susceptibility of lactobacilli against two antifungal drugs: isoconazole and oxiconazole. It was concluded that lactobacilli harbour a natural resistance against these drugs. Our findings affirmed this conclusion. L. fermentum Ess-1 was resistant to all antifungal drugs tested, except for flucytosine $(0.125 \mu \mathrm{g}$ $\left.\mathrm{ml}^{-1}\right)$. C. glabrata harbours a relatively low susceptibility to azoles in vitro but shows high susceptibility to flucytosine (Richter et al., 2005). The first report of intravaginal flucytocine treatment for women positive for C. glabrata showed a $90 \%$ cure rate (Sobel et al., 2003). However, long-term use of flucytocine to prevent reinfection or relapse was not recommended. At this step, an intravaginal supply of $L$. fermentum Ess-1 could be a promising intervention to prevent $C$. glabrata overgrowth in the vulva and vagina. For this purpose, it is of great importance that L. fermentum Ess-1 survives and stays viable when supplied to the vagina. A pilot study was carried out with vaginal insertion of L. fermentum Ess-1. Four volunteers inserted capsules containing a lyophilized powder of $L$. fermentum Ess- 1 the week prior to menses. $L$. fermentum Ess-1 was detected in all of the women after menses and the mean number of identified $L$. fermentum Ess-1 was $3.7 \log _{10}$ c.f.u. per sample. The samples with the highest and lowest numbers contained 6.2 and $1.3 \log _{10}$ c.f.u. per sample, respectively. These results strengthen the potential use of this strain as a vaginal probiotic. Further tests showed that the bacterial number in the lyophilized powder was reduced from 10.0 to $8.9 \log _{10}$ c.f.u. $g^{-1}$ after 11 weeks of storage in sealed tubes at $37^{\circ} \mathrm{C}$. Survival at room temperature is believed to be considerably longer, which would facilitate the distribution and storage of sealed products containing L. fermentum Ess-1. Future studies to evaluate the possible clinical benefits of $L$.

Table 1. Inhibition scores for LCF against C. albicans CCUG 44135 at three different $\mathrm{pH}$ levels

Growth inhibition was scored from 0 (no visual inhibition) to 10 (complete visual inhibition), as shown in Fig. 1. SD was $\pm 0-0.41$.

\begin{tabular}{|ccccc|}
\hline pH & Ess-1 & LB99 & LB931 & dMRSs \\
\hline 3.7 & 8.5 & 2 & 0 & 0 \\
5.0 & 7.5 & 2 & 0 & 0 \\
7.0 & 8 & 2 & 0.5 & 0 \\
\hline
\end{tabular}

fermentum Ess-1 in women with VVC are essential and have been initiated.

\section{Conclusion}

Metabolites produced by L. fermentum Ess-1 show exceptional fungistatic properties against the two most common yeast species associated with VVC, C. albicans and C. glabrata. L. fermentum Ess-1 has great potential to be used as a probiotic to treat symptomatic VVC or to prevent recurrent VVC infection.

\section{ACKNOWLEDGEMENTS}

We are grateful to Francesca Valerio, Institute of Sciences of Food Production, Bari, Italy, for providing some of the reference strains. We also thank Rolf Andersson and Stellan Håkansson for comments on the manuscript.

\section{REFERENCES}

Demirezen, S. (2002). The Lactobacilli-Candida relationship in cervico-vaginal smears. Cent Eur J Public Health 10, 97-99.

Juarez Tomas, M. S., Ocana, V. S., Wiese, B. \& Nader-Macias, M. E. (2003). Growth and lactic acid production by vaginal Lactobacillus acidophilus CRL 1259, and inhibition of uropathogenic Escherichia coli. J Med Microbiol 52, 1117-1124.

Kaewsrichan, J., Peeyananjarassri, K. \& Kongprasertkit, J. (2006). Selection and identification of anaerobic lactobacilli producing inhibitory compounds against vaginal pathogens. FEMS Immunol Med Microbiol 48, 75-83.

Kilic, E., Aslim, B. \& Taner, Z. (2005). Susceptibility to some antifungal drugs of vaginal lactobacilli isolated from healthy women. Drug Metabol Drug Interact 21, 67-74.

Martens, M. G., Hoffman, P. \& El-Zaatari, M. (2004). Fungal species changes in the female genital tract. J Low Genit Tract Dis 8, 21-24.

Okkers, D. J., Dicks, L. M., Silvester, M., Joubert, J. J. \& Odendaal, H. J. (1999). Characterization of pentocin TV35b, a bacteriocin-like peptide isolated from Lactobacillus pentosus with a fungistatic effect on Candida albicans. J Appl Microbiol 87, 726-734.

Paulitsch, A., Weger, W., Ginter-Hanselmayer, G., Marth, E. \& Buzina, W. (2006). A 5-year (2000-2004) epidemiological survey of Candida and non-Candida yeast species causing vulvovaginal candidiasis in Graz, Austria. Mycoses 49, 471-475.

Reid, G. \& Bruce, A. W. (2003). Urogenital infections in women: can probiotics help? Postgrad Med J 79, 428-432.

Richter, S. S., Galask, R. P., Messer, S. A., Hollis, R. J., Diekema, D. J. \& Pfaller, M. A. (2005). Antifungal susceptibilities of Candida species causing vulvovaginitis and epidemiology of recurrent cases. J Clin Microbiol 43, 2155-2162.

Rönnqvist, D., Strom, H., Forsgren-Brusk, U. \& Grahn-Hakansson, E. (2005). Selection and characterization of a Lactobacillus plantarum strain promising as a urogenital probiotic. Microb Ecol Health Dis 17, 75-82.

Runeman, B., Rybo, G., Forsgren-Brusk, U., Larko, O., Larsson, P. \& Faergemann, J. (2004). The vulvar skin microenvironment: influence of different panty liners on temperature, $\mathrm{pH}$ and microflora. Acta Derm Venereol 84, 277-284. 
Sobel, J. D. \& Chaim, W. (1996). Vaginal microbiology of women with acute recurrent vulvovaginal candidiasis. J Clin Microbiol 34, 2497-2499.

Sobel, J. D., Chaim, W., Nagappan, V. \& Leaman, D. (2003). Treatment of vaginitis caused by Candida glabrata: use of topical boric acid and flucytosine. Am J Obstet Gynecol 189, 1297-1300.

Spinillo, A., Capuzzo, E., Gulminetti, R., Marone, P., Colonna, L. \& Piazzi, G. (1997). Prevalence of and risk factors for fungal vaginitis caused by non-albicans species. Am J Obstet Gynecol 176, 138-141.

Stiles, J., Penkar, S., Plockova, M., Chumchalova, J. \& Bullerman, L. B. (2002). Antifungal activity of sodium acetate and Lactobacillus rhamnosus. J Food Prot 65, 1188-1191.

Strus, M., Brzychczy-Wloch, M., Kucharska, A., Gosiewski, T. \& Heczko, P. B. (2005a). Inhibitory activity of vaginal Lactobacillus bacteria on yeasts causing vulvovaginal candidiasis. Med Dosw Mikrobiol 57, 7-17.
Strus, M., Kucharska, A., Kukla, G., Brzychczy-Wloch, M., Maresz, K. \& Heczko, P. B. (2005b). The in vitro activity of vaginal Lactobacillus with probiotic properties against Candida. Infect Dis Obstet Gynecol 13, 69-75.

Vasquez, A., Jakobsson, T., Ahrne, S., Forsum, U. \& Molin, G. (2002). Vaginal lactobacillus flora of healthy Swedish women. J Clin Microbiol 40, 2746-2749.

Voravuthikunchai, S. P., Bilasoi, S. \& Supamala, O. (2006). Antagonistic activity against pathogenic bacteria by human vaginal lactobacilli. Anaerobe 12, 221-226.

Wilson, J. (2004). Managing recurrent bacterial vaginosis. Sex Transm Infect 80, 8-11.

Wynne, A. G., McCartney, A. L., Brostoff, J., Hudspith, B. N. \& Gibson, G. R. (2004). An in vitro assessment of the effects of broad-spectrum antibiotics on the human gut microflora and concomitant isolation of a Lactobacillus plantarum with anti-Candida activities. Anaerobe 10, $165-169$. 